\title{
Outage Performance of Relay-Assisted Single- and Dual-Stage NOMA Over Power Line Communications
}

\author{
ROOPESH RAMESH ${ }^{1}$, (Graduate Student Member, IEEE), SANJEEV GURUGOPINATH ${ }^{\circledR}$, \\ (Member, IEEE), AND SAMI MUHAIDAT ${ }^{\circledR 2}$, (Senior Member, IEEE) \\ ${ }^{1}$ Department of Electronics and Communication Engineering, PES University, Bengaluru 560085, India \\ ${ }^{2}$ Center for Cyber-Physical Systems, Department of Electrical and Computer Engineering, Khalifa University, Abu Dhabi, United Arab Emirates \\ Corresponding Author: Sami Muhaidat (muhaidat@ieee.org)
}

\begin{abstract}
In this paper, we analyze the performance of relay-assisted, single-stage (SS) non-orthogonal multiple access (NOMA) and dual-stage (DS) NOMA power line communication systems. Specifically, we derive closed form expressions for the outage probabilities of the SS NOMA and DS NOMA schemes. Subsequently, we formulate optimization problems and obtain closed-form solutions for the optimal power allocation coefficients of the SS NOMA and DS NOMA schemes, such that the probability of overall outage is minimized. The accuracy of our analysis and the tightness of the approximations employed are validated through Monte Carlo simulations and numerical techniques. Moreover, we show that the DS NOMA scheme outperforms the SS NOMA scheme, in terms of the overall outage probability.
\end{abstract}

INDEX TERMS Cooperative relaying, outage probability analysis, non-orthogonal multiple access (NOMA), power line communication (PLC), single- and dual-stage NOMA.

\section{INTRODUCTION}

\section{A. BACKGROUND}

The simplicity of utilizing pre-existing wired infrastructure makes power-line communications (PLC) a promising technology for broadband and narrowband communications, especially for applications such as smart grids and home automation. This efficient utilization of the existing infrastructure reduces the deployment cost significantly. The narrowband PLC technology is useful, where an arrangement of bidirectional correspondence interconnecting hubs, is a requirement, in applications such as smart grids [2]. In particular, PLC is envisioned over the low voltage (LV) and medium voltage (MV) lines, which carry power and data simultaneously, with little/negligible interference. On the other hand, wideband PLC provides solutions to applications such as home-networking, internet-of-things, and also some use cases in smart grids. Another attractive feature of PLC networks is the high data rate due to the wired communication, where the information does not undergo significant degradation, in contrast to wireless communications.

The associate editor coordinating the review of this manuscript and approving it for publication was Chi-Hua Chen ${ }^{\text {(D) }}$.
Additionally, PLC also helps in achieving a ubiquitous coverage - e.g., as a backhaul communication link in wireless networks - as an alternative to complement existing technologies. Moreover, thanks to the recent advancements in signal processing for communications techniques, secure communication over PLC can also be achieved, even though PLC does not exhibit a favorable communication medium in contrast to optical fiber and the wireless medium [3]-[5]. Aspects such as security, robustness and high-data rates makes PLC as an attractive technology for design of smart grid, in contrast to wireless technologies [6]. Reliability is an additional advantage in PLC for indoor communications, since Wi-Fi signals experience degradation due to concrete and metal walls [7]-[9]. The reliability can be further enhanced by ensuring an electromagnetic compatible design [10], which also boosts the data rates further [1], [11], [12].

However, attenuation, channel impedance, frequencyselective multipath propagation and receiver noise in PLC systems introduce random fluctuations in the transmitted signals over time and frequency [11], [13], [14]. Additional impairments are due to discontinuities, unmatched load and

This article was presented in part at the Proc. PIMRC 2020 [1]. 
52 receiver location [15]. Moreover, the presence of surround53 ing wireless communication systems create electromagnetic 54 interference to PLC systems, which is another major challenge [16]. These impairments introduce several challenges in modeling PLC channels [17]. Performance losses due to the presence of frequency-selective fading and non-Gaussian noise [18] can be mitigated by employing multi-carrier modulation schemes such as orthogonal frequency division multiplexing (OFDM) [11], [19]-[21]. Additionally, relay-based schemes such as the amplify-and-forward (AF) and decodeand-forward (DF) techniques are employed to alleviate the aforementioned issues, under transmit power constraints [22], [23]. In the conventional relay-based cooperative PLC systems based on orthogonal multiple access (OMA) techniques such as time division multiplexing access (TDMA) and OFDM, the receiver combines the signal from the transmitter with a copy from the relay over two time slots [24]-[27]. This leads to a degradation of the overall spectral efficiency, even though the improved performance at the receiver is enhanced in terms of the bit error rate.

Non-orthogonal multiple access (NOMA) is a promising technology that overcomes the disadvantages due to OMA at the expense of extra computational complexity [28], [29]. A growing body of research shows that NOMA is suitable for the fifth generation and beyond $(5 \mathrm{G}+)$ communication systems, since it offers a higher data rate and system capacity in contrast to the preceding OMA technologies such as TDMA and OFDM [30], [31]. An attractive feature of NOMA is that every user is allowed to occupy the same time/frequency/space resource, and users are multiplexed in either the power-domain (PD) or code-domain (CD). In PD NOMA (simply called NOMA hereon), the source transmits employs the principle of superposition to combine the information corresponding to different users, with an appropriately designed power allocation policy [32], [33]. In other words, a NOMA transmitter can simultaneously transmit multiple user signals with different power levels, using the entire frequency at the same time. At the receiver, each user employs a non-linear decoding technique, known as the successive interference cancellation (SIC), to separate the information from the multiplexed signal and to decode its own signal [34]. A considerable difference in power levels across the users is maintained at the transmitter to establish a low error demultiplexing of user signals at the receiver using SIC [30], [34]. It is known that the NOMA offers greater achievable rate regions compared to OMA techniques such as TDMA and OFDMA, as long as users are assigned with different power levels [33], [35], [36]. Theoretically, employing NOMA is optimal in terms of achieving the boundaries of multi-user capacity [22], [37]. The main idea in cooperative NOMA is to utilize the near user as a relay to enhance the performance of the far user. As opposed to the co-operative relaying considering energy efficiency issues, simultaneous wireless information and power transfer (SWIPT) was employed at the near user in [38] for a wireless scenario, which was equivalent to DF relaying. Cooperative NOMA with full-duplex relaying helps in enhancing the performance gain of the far user, as shown in [39]. It is also expected that the users with better channel conditions naturally benefit more NOMA as opposed to the users with worse channel conditions due to high interference. This attractive feature enables NOMA to be compatible with several other existing technologies. Overall, NOMAbased cooperative relaying offers the following advantages, compared to a OMA-based relaying system. First, it offers a higher system throughput, due to simultaneous transmission of data to different users. Second, it ensures better fairness among users by serving multiple users for each channel use. Moreover, it helps in mitigating the impact of harshness and hostility in a PLC environment on its performance, and offers considerable improvements in spectral efficiency and range extension [40]. Furthermore, it also helps in the relaxing the electromagnetic compatibility constraints, which results in a better coexistence with other wireless networks [41].

\section{B. MOTIVATION}

Given the aforementioned advantages of NOMA, the idea of non-orthogonal transmission can be employed in a relayaided PLC network, with a combination of superposition coding (SC) at the transmitter PLC modem and SIC at the relay and receiver PLC modems [12]. Employing SC at a NOMA PLC transmitter allows multiple users with varying power levels to be served simultaneously in the same time, frequency, and space resource block, which increases the system throughput. Since the superimposed signal from the transmitter modem contains information corresponding to different users, the overall transmit power reduces, which helps in mitigating the electromagnetic compatibility problem in PLC, particularly, in applications such as smart grids where different users communicate with different data rates, relay-aided NOMA PLC provides high spectral efficiency and fairness across users. Motivated by these advantages, the performance of NOMA in relay-based PLC was recently proposed and studied in [12], [32], where the authors showed that the NOMA-based PLC network improves the achievable capacity, as compared to conventional OMA-based cooperative communication. However, the analysis in [32] is limited to the achievable capacity, and does not provide a closed-form expression for the derived capacity. Additionally, the reported results do not provide theoretical insights into the optimal power allocation at the transmitter modem, which is an important design metric in NOMA.

In this work, we consider a NOMA-based PLC system similar to the setup described in [32] with two different schemes, namely the single-stage NOMA and dual-stage NOMA [41]. In the single-stage scheme, the relay employs a DF approach and forwards the decoded signal to the receiver which combines the two copies of by employing the maximal ratio combining [22], [23]. On the other hand, in contrast to the single-stage scheme, the destination modem in a dual-stage scheme does not decode the received signal from the source until it receives the signals from the two time slots and then 
163 jointly decodes the two symbols with equal gain combining, 64 which enhances the performance of the PLC network [41]. There are two main advantages with this scheme. First, using the principle of SC in NOMA allows for a better utilization of transmit power at the relay. Second, this scheme reduces the outage probability due to user ordering, and enhances the overall spectral efficiency of the PLC network, which enables accommodation of more number of users. As opposed to the works in [32] and [41], we consider the probability of outage as a the performance metric, and provide a detailed analysis on the power allocation policy at the transmitter modem for the single-stage scheme and the power allocation policies at the transmitter and relay modems for the dual-stage scheme. Moreover, we consider the performance of single- and dualstage relay-based PLC systems in this work, as opposed to [38] and [39].

\section{CONTRIBUTIONS}

We consider a relay-assisted PLC system with a source, a relay and a destination with two schemes namely (a) singlestage (SS) NOMA, where the principle of superposition is employed at the source to convey information to both relay and destination, and (b) dual-stage (DS) NOMA, where superposition coding is also employed at the relay along with the source to enhance the decoding performance at the destination. In particular, we study the outage probability performance of the considered system, and derive approximate closed form solutions. Next, we find the optimal power allocation policy at the (a) source for SS NOMA and (b) source and relay for DS NOMA, such that the corresponding overall outage probability is minimized, in contrast to the works reported in [32], [38], [39] and [41]. We present an extensive set of Monte Carlo and numerical techniques-based results to validate our analysis, and to establish that the DS NOMA offers a better performance than SS NOMA in terms of outage probability. The main contributions of this paper can be summarized as below.

- We consider the SS NOMA and DS NOMA schemes for a relay-assisted PLC, and derive the expressions for the probabilities of overall outage at the relay and destination.

- For both SS NOMA and DS NOMA schemes, we formulate optimization problems to find the optimal power allocation at the source and relay, such that the overall outage probabilities are minimized.

- We derive closed form expressions for the optimal power allocation coefficients at the source for SS NOMA and at source and relay for DS NOMA. We show that these optimal power allocation coefficients are independent of the system parameters such as the operating frequency and distance between the PLC modems.

- Through numerical results and Monte Carlo simulations, we validate the tightness of some approximations used in our analysis for the derivation of outage probability. Additionally, we provide insights into the impact of the PLC system parameters such as the dis- tance and fading parameters on the outage performance. Furthermore, we show that the DS NOMA scheme, as expected, gives a better performance compared to the SS NOMA scheme.

\section{ORGANIZATION}

The remainder of the paper is organized as follows. We describe the system model, and SS NOMA and DS NOMA schemes in Section II. We derive expressions for the outage probabilities at the relay and the destination for both SS NOMA and DS NOMA schemes in Section III. In Section IV, we formulate optimization problems to find optimal power coefficients at the source modem for SS NOMA and DS NOMA schemes, such that the overall outage probabilities at the relay and destination are minimized. Our analysis is validated through Monte Carlo simulations and numerical technique in Section $\mathrm{V}$, which also provides more insights into the considered NOMA-PLC networks. Finally, we provide concluding remarks in Section VI.

\section{SYSTEM MODEL}

Fig. 1 illustrates our system model, which consists of one source (S) PLC modem and two receiver PLC modems. The receiver modem nearer to $S$ also acts as a relay $(R)$ and the other one is the destination (D), which is farther from $\mathrm{S}$ compared to $\mathrm{R}$. It is assumed that channel state information is perfectly known at both the receiver modems. The channel gains between S-R, S-D and R-D, denoted by $g_{S R}, g_{S D}$, and $g_{R D}$, respectively, are modeled as independent and identically distributed (i.i.d.) lognormal random variables [42], [43]. The lognormal fading model in PLC systems characterizes the statistical effects introduced by the load mismatch in the PLC network topology and the signal propagation through the network branches [44]. The PDFs of these channel gains are given by

$$
f\left(g_{i}\right)=\frac{1}{\sqrt{2 \pi} \sigma_{i} g_{i}} \exp \left[-\frac{\left(\log g_{i}-\mu\right)^{2}}{2 \sigma^{2}}\right],
$$

for $i \in\{\mathrm{SR}, \mathrm{SD}, \mathrm{RD}\}$. Here $\mu$ and $\sigma^{2}$ denote the mean and the variance of $10 \log _{10}\left(g_{i}\right), i \in\{\mathrm{SR}, \mathrm{SD}, \mathrm{RD}\}$ respectively. We denote the distribution of $g_{i}$ as $g_{i} \sim \mathcal{L} \mathcal{N}\left(\mu_{i}, \sigma_{i}^{2}\right), i \in$ $\{\mathrm{SR}, \mathrm{SD}, \mathrm{RD}\}$. Additionally, we also consider the distanceand frequency-dependent channel attenuation, which are denoted by $G_{i}\left(v_{i}, f\right)$ for $i \in\{\mathrm{SR}, \mathrm{SD}, \mathrm{RD}\}$, respectively, where $v_{S R}, v_{S D}$ and $v_{R D}$ are the distances between the S-R, S-D and R-D, and $f$ is the frequency of communication. Let $P$ denote the total power available at $\mathrm{S}$. The communication from $\mathrm{S}$ to $\mathrm{R}$ and $\mathrm{D}$ is accomplished over two time slots, with the goal to transmit two symbols $s_{1}$ and $s_{2}$ to both receivers, that is, to both $\mathrm{R}$ and $\mathrm{D}$. The transmission scheme employed by $\mathrm{S}$ uses the superposition coding principle following powerdomain NOMA [45]. We consider two different transmission schemes for the communication from $\mathrm{S}$ to $\mathrm{R}$ and D. First, we consider the SS NOMA, where the superposed signal is transmitted only in the first time slot. Secondly, we consider DS NOMA, wherein the superposition coding is also used 


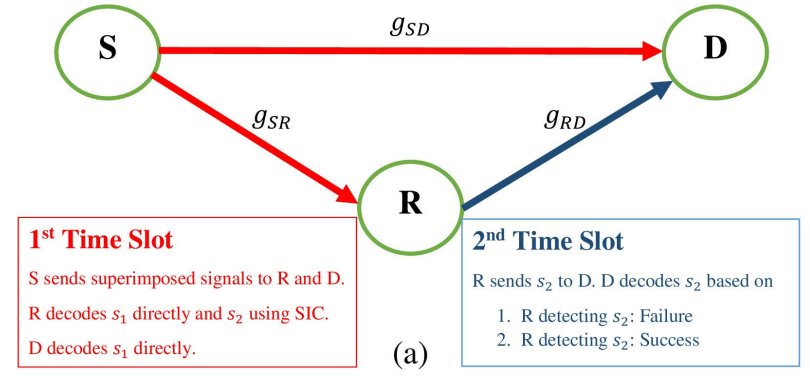

(a)

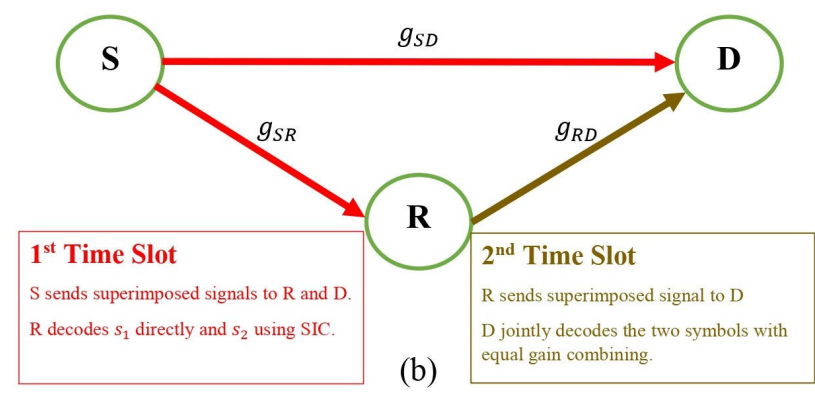

FIGURE 1. System model and time slots description for (a) SS NOMA, and (b) DS NOMA.

301 respectively.

\footnotetext{
${ }^{1}$ This assumption simplifies our analysis. The implication of relaxing this assumption is discussed in detail in Section III.
}

respectively. Here, $w_{R}$ and $w_{D}$ denote the noise samples at the $\mathrm{R}$ and $\mathrm{D}$, respectively, which are modeled as independent, zero-mean Gaussian random variables with variances $\sigma_{R}^{2}$ and $\sigma_{D}^{2}$, respectively. That is, $w_{R} \sim \mathcal{N}\left(0, \sigma_{R}^{2}\right)$ and $w_{D} \sim \mathcal{N}\left(0, \sigma_{D}^{2}\right)$, independent of each other.

At the end of the first time slot, the signals $s_{1}$ and $s_{2}$ are decoded at $\mathrm{R}$ as follows. Signal $s_{1}$ is decoded directly by considering the interference due to $s_{2}$ as noise, and the signal $s_{2}$ is decoded using the successive interference cancellation (SIC). To calculate the SINR values at $\mathrm{R}$, we assume perfect SIC, i.e., the interference due to $s_{1}$ is completely removed before decoding $s_{2} .{ }^{1}$ Therefore, the SINR at R from the S-R link for decoding $s_{1}$ and $s_{2}$ are given by

$$
\begin{aligned}
& \zeta_{S}^{\left(s_{1}\right)} \triangleq \frac{b_{1} P\left[G_{S R}\left(v_{S R}, f\right)\right]^{2} g_{S R}^{2}}{b_{2} P\left[G_{S R}\left(v_{S R}, f\right)\right]^{2} g_{S R}^{2}+\sigma_{R}^{2}}, \quad \text { and } \\
& \zeta_{S}^{\left(s_{2}\right)} \triangleq \frac{b_{2} P\left[G_{S R}\left(v_{S R}, f\right)\right]^{2} g_{S R}^{2}}{\sigma_{R}^{2}},
\end{aligned}
$$

Decoding at D differs for SS NOMA and DS NOMA schemes. In SS NOMA, the signal $s_{1}$ is decoded at D directly by treating the interference due to $s_{2}$ as noise. Therefore, the SINR at $\mathrm{D}$ for decoding $s_{1}$ is given by

$$
\zeta_{D}^{\left(s_{1}\right)}=\frac{b_{1} P\left[G_{S D}\left(v_{S D}, f\right)\right]^{2} g_{S D}^{2}}{b_{2} P\left[G_{S D}\left(v_{S D}, f\right)\right]^{2} g_{S D}^{2}+\sigma_{D}^{2}} .
$$

We assume that $\mathrm{D}$ decodes $s_{1}$ perfectly, since $b_{1}>b_{2}$ [32]. In the case of DS NOMA, D jointly decodes the two symbols over two time slots, which is explained in Section II-B2.

\section{B. SECOND TIME SLOT}

1) SS NOMA

In SS NOMA, R forwards the decoded signal $s_{2}$ to D during the second time slot. Let $P_{R}$ be the power available at $\mathrm{R}$ for the transmission. Then, the relay transmits

$$
x_{2}=\sqrt{P_{R}} s_{2}
$$

to $\mathrm{D}$. Depending on whether $\mathrm{R}$ was able to decode $s_{2}$ or not in the first time slot, the following two cases $(\mathrm{C} 1$ and $\mathrm{C} 2)$ occur in the second time slot.

C1. If $s_{2}$ was not successfully decoded by R, the information at $\mathrm{D}$ is available only from the received signal $z_{D}$ from the first time slot. Therefore, D tries to decode $s_{2}$ directly from (4). In this case, the received SINR at D is given by

$$
\zeta_{D}^{\left(s_{2}, 1\right)}=\frac{b_{2} P\left[G_{S D}\left(v_{S D}, f\right)\right]^{2} g_{S D}^{2}}{b_{1} P\left[G_{S D}\left(v_{S D}, f\right)\right]^{2} g_{S D}^{2}+\sigma_{D}^{2}} .
$$

C2. If $s_{2}$ was successfully decoded by R, then the received signal at $\mathrm{D}$ is given by

$$
z_{R D}=x_{2} G_{R D}\left(v_{R D}, f\right) g_{R D}+w_{D}^{\prime},
$$

where $w_{D}^{\prime} \sim \mathcal{N}\left(0, \sigma_{D}^{2}\right)$. Now, D uses the maximum ratio combining (MRC) to combine the received signals from both time slots with coefficients $\omega_{1}$ and $\omega_{2}$, which yields the total signal

$$
z_{c}=\omega_{1} z_{D}+\omega_{2} z_{R D} .
$$




\section{2) DS NOMA}

37 The SS NOMA scheme uses the principle of NOMA only during the first time slot, and employs the MRC at the D. The usage of MRC requires the symbol $s_{2}$ to be decoded successfully at the relay, which is allocated with lower power. The probability of decoding the symbol $s_{2}$ directly affects the outage probability of the network. In order to overcome this problem and to improve the performance of the system, DS NOMA approach is considered, where the superposition coding is also employed in the second time slot. In DS NOMA, the signal which was sent from $\mathrm{S}$ to $\mathrm{D}$ during the first time slot is not individually decoded, but it jointly decodes the signals after the second time slot. In the second slot, the relay forwards the superposed signal,

$$
x_{3} \triangleq\left(\sqrt{b_{3} P} s_{1}-\sqrt{b_{4} P} s_{2}\right)
$$

to $\mathrm{D}$, using the power allocation coefficients $b_{3}$ and $b_{4}$, where $b_{3}+b_{4}=1$. It should be noted that the minus sign in (13) is necessary, which - as will be shown in the sequel - can help us perfectly cancel the interference signals at D. With (13), the received signal at $\mathrm{D}$ in the second time slot is given by

$$
z_{D}^{(I I)}=x_{3} G_{R D}\left(v_{R D}, f\right) g_{R D}+w_{D} .
$$

Now, for DS NOMA, two signals $z_{D}$ and $z_{D}^{(I I)}$ are available at D vide (4) and (14), from which the signals $s_{1}$ and $s_{2}$ are jointly decoded by employing the following linear combinations

$$
\begin{aligned}
& s_{D}^{(1)}=z_{D} \sqrt{b_{4}} g_{R D}+z_{D}^{(I I)} \sqrt{b_{2}} g_{S D} \\
& s_{D}^{(2)}=z_{D} \sqrt{b_{3}} g_{R D}-z_{D}^{(I I)} \sqrt{b_{1}} g_{S D} .
\end{aligned}
$$

Substituting for $z_{D}$ and $z_{D}^{(I I)}$, the signals $s_{1}$ and $s_{2}$ decoded at the $\mathrm{D}$ can be obtained from

$$
\begin{aligned}
s_{D}^{(1)}= & \left(\sqrt{b_{1} b_{4} P} s_{1}+\sqrt{b_{2} b_{4} P} s_{2}\right) G_{S D} g_{S D} G_{R D} g_{R D} \\
& +\left(\sqrt{b_{3} b_{2} P_{R}} s_{1}-\sqrt{b_{4} b_{2} P_{R}} s_{2}\right) G_{S D} g_{S D} G_{R D} g_{R D} \\
& +\sqrt{b_{4}} G_{R D} g_{R D} w_{D}+\sqrt{b_{2}} G_{S D} g_{S D} w_{D} . \\
s_{D}^{(2)}= & \left(\sqrt{b_{1} b_{3} P} s_{1}+\sqrt{b_{2} b_{3} P} s_{2}\right) G_{S D} g_{S D} G_{R D} g_{R D} \\
& -\left(\sqrt{b_{3} b_{1} P_{R}} s_{1}-\sqrt{b_{4} b_{1} P_{R}} s_{2}\right) G_{S D} g_{S D} G_{R D} g_{R D} \\
& +\sqrt{b_{3}} G_{R D} g_{R D} w_{D}+\sqrt{b_{1}} G_{S D} g_{S D} w_{D} .
\end{aligned}
$$

For the ease of analysis, we assume that $P=P_{R}, \sigma_{R}^{2}=$ $\sigma_{D}^{2}=\sigma^{2}$ and let $\rho=P / \sigma^{2}$. Substituting these, we get

$$
\begin{aligned}
s_{D}^{(1)}= & G_{S D} g_{S D} G_{R D} g_{R D} \eta \sqrt{P} s_{1} \\
& +\sqrt{b_{4}} G_{R D} g_{R D} w_{D}+\sqrt{b_{2}} G_{S D} g_{S D} w_{D}, \\
s_{D}^{(2)}= & G_{S D} g_{S D} G_{R D} g_{R D} \eta \sqrt{P} s_{2}
\end{aligned}
$$

$$
+\sqrt{b_{3}} G_{R D} g_{R D} w_{D}+\sqrt{b_{1}} G_{S D} g_{S D} w_{D},
$$

where $\eta=\sqrt{b_{1} b_{4}}+\sqrt{b_{2} b_{3}}$. Following this, one can perform symbol-by-symbol detection to decode $s_{1}$ and $s b$, and the corresponding SINR values at D can be expressed as

$$
\begin{aligned}
\zeta_{D}^{(I)} & =\frac{\prod_{i \in\{\mathrm{SD}, \mathrm{RD}\}} G_{i}^{2} g_{i}^{2} \eta^{2} \rho}{b_{4} G_{R D}^{2} g_{R D}^{2}+b_{2} G_{S D}^{2} g_{S D}^{2}}, \\
\zeta_{D}^{(I I)} & =\frac{\prod_{i \in\{\mathrm{SD}, \mathrm{RD}\}} G_{i}^{2} g_{i}^{2} \eta^{2} \rho}{b_{3} G_{R D}^{2} g_{R D}^{2}+b_{1} G_{S D}^{2} g_{S D}^{2}} .
\end{aligned}
$$

It should be noted that while the SINR values at $\mathrm{R}$ for both SS NOMA and DS NOMA schemes are equal, the corresponding SINR values at D are different. As mentioned earlier, employing NOMA for PLC allows the transmission of two symbols $s_{1}$ and $s_{2}$ over two time slots, and, hence, offers better spectral efficiency than any other OMA scheme. In the next section, we provide an analysis on the overall outage probability for the considered system model, with SS NOMA and DS NOMA.

\section{OUTAGE ANALYSIS}

Let the thresholds on SINRs for a given requirements on the data rate at both $\mathrm{R}$ and $\mathrm{D}$ for symbols $s_{1}$ and $s_{2}$ be denoted by $\gamma_{1}$ and $\gamma_{2}$, respectively. First, the outage probability at $R$ is expressed as

$$
\begin{aligned}
P_{\text {out }}^{(R)} & \triangleq \operatorname{Pr}\left\{\zeta_{S}^{\left(s_{1}\right)} \leq \gamma_{1} \text { or } \zeta_{S}^{\left(s_{2}\right)} \leq \gamma_{2}\right\}, \\
& =\operatorname{Pr}\left\{P\left[G_{S R}\left(v_{S R}, f\right)\right]^{2} g_{S R}^{2} \leq \Gamma\right\},
\end{aligned}
$$

where $\Gamma \triangleq \max \left(\frac{\gamma_{1} \sigma_{R}^{2}}{b_{1}-b_{2} \gamma_{1}}, \frac{\gamma_{2} \sigma_{R}^{2}}{b_{2}}\right)$. From the properties of the lognormal distribution,

$$
\begin{aligned}
\text { if } g_{S R} & \sim \mathcal{L N}\left(\mu, \sigma^{2}\right), \\
\text { then } g_{S R}^{2} & \sim \mathcal{L N}\left(2 \mu, 4 \sigma^{2}\right), \\
\text { and } a g_{S R} & \sim \mathcal{L N}\left(\mu+\log a, \sigma^{2}\right), \quad \forall a \in \mathbb{R}^{+} .
\end{aligned}
$$

Following these properties, it is straight forward that the outage probability at $\mathrm{R}$ can be shown as

$$
P_{\mathrm{out}}^{(R)}=\frac{1}{2} \operatorname{erfc}\left(-\frac{\log (\Gamma)-\left(2 \mu+\log \left(P\left[G_{S R}\left(v_{S R}, f\right)\right]^{2}\right)\right)}{\sqrt{8} \sigma}\right),
$$

where $\operatorname{erfc}(\cdot)$ is the complementary error function [32]. Next, we consider the outage analysis at D for SS NOMA and DS NOMA separately.

\section{A. SS NOMA}

1) PERFECT DECODING OF $s_{1}$ AT D

First, we continue with our assumption that D perfectly decodes $s_{1}$ in the first time slot [32]. Therefore, the outage probability at $\mathrm{D}$ for the two cases $\mathrm{C} 1$ and $\mathrm{C} 2$ are defined as

$$
\mathrm{C} 1 .: P_{\mathrm{out}}^{(D)} \triangleq \operatorname{Pr}\left\{\zeta_{D}^{\left(s_{2}, 1\right)} \leq \gamma_{2}\right\}
$$




$$
\text { C2. : } P_{\text {out }}^{(D)} \triangleq \operatorname{Pr}\left\{\zeta_{D}^{\left(s_{2}, 2\right)} \leq \gamma_{2}\right\} \text {. }
$$

For $\mathrm{C} 1$, recall that the SINR at D is given by $\zeta_{D}^{(1)}$ in (9). Therefore, the outage probability in this case is given as

$$
\begin{aligned}
P_{\mathrm{out}}^{(D, 1)} & =\operatorname{Pr}\left\{\zeta_{D}^{\left(s_{2}, 1\right)} \leq \gamma_{2}\right\} \\
& =\operatorname{Pr}\left\{\mathcal{K} P\left[G_{S D}\left(v_{S D}, f\right)\right]^{2} g_{S D}^{2} \leq \gamma_{2} \sigma_{D}^{2}\right\},
\end{aligned}
$$

where $\mathcal{K} \triangleq\left(b_{2}-b_{1} \gamma_{2}\right)$. Following the properties of lognormal distribution discussed in (25)-(27) that lead to (28), we get

$$
\begin{aligned}
P_{\text {out }}^{(D, 1)}=\frac{1}{2} \operatorname{erfc}( & -\frac{\log \left(\gamma_{2} \sigma_{d}^{2}\right)}{\sqrt{8} \sigma} \\
& \left.+\frac{\left(2 \mu+\log \left(P\left[G_{S D}\left(v_{S D}, f\right)\right]^{2} \mathcal{K}\right)\right)}{\sqrt{8} \sigma}\right) .
\end{aligned}
$$

Next, for $\mathrm{C} 2$, wherein the symbol $s_{2}$ is successfully detected by $\mathrm{R}$, the SINR at $\mathrm{D}$ is given by $\zeta_{D}^{\left(s_{2}, 2\right)}$ in $(12)$ Therefore,

$$
\begin{aligned}
P_{\text {out }}^{(D, 2)} & =\operatorname{Pr}\left\{\zeta_{D}^{\left(s_{2}, 2\right)} \leq \gamma_{2}\right\} \\
& =\operatorname{Pr}\left\{X+Y \leq \gamma_{2}\right\},
\end{aligned}
$$

where

$$
\begin{aligned}
& X \triangleq \frac{b_{2} P\left[G_{S D}\left(v_{S D}, f\right)\right]^{2} g_{S D}^{2}}{b_{1} P\left[G_{S D}\left(v_{S D}, f\right)\right]^{2} g_{S D}^{2} \sigma_{D}^{2}}, \\
& Y \triangleq \frac{P_{R}\left[G_{R D}\left(v_{R D}, f\right)\right]^{2} g_{R D}^{2}}{\sigma_{D}^{2}} .
\end{aligned}
$$

Obtaining a closed form solution for the CDF in (35) ${ }_{435}$ is hard. Towards this end, we consider the following two36 tier approximation on the distribution of random variables. We show the tightness of this approximation later in Section V. Following the two approximations, (35) can be written as

$$
\begin{aligned}
P_{\mathrm{out}}^{(D)} & =\operatorname{Pr}\left\{X+Y \leq \gamma_{2}\right\} \\
& \stackrel{(a)}{\approx} \operatorname{Pr}\left\{X^{\prime}+Y \leq \gamma_{2}\right\}, \\
& \stackrel{(b)}{\approx} \operatorname{Pr}\left\{Z \leq \gamma_{2}\right\},
\end{aligned}
$$

where, in the first approximation denoted by $(a)$ in (39), the new random variable $X^{\prime}$ is obtained by replacing $g_{S D}^{2}$ in the denominator of (36) by its expected value, that is $\mathbb{E} g_{S D}^{2}=e^{2 \mu+2 \sigma^{2}}$. That is,

$$
X^{\prime} \triangleq \frac{b_{2} P\left[G_{S D}\left(f, v_{S D}\right)\right]^{2} g_{S D}^{2}}{b_{1} P\left[G_{S D}\left(v_{S D}, f\right)\right]^{2}\left(e^{2 \mu+2 \sigma^{2}}\right) \sigma_{D}^{2}} .
$$

As the second approximation, denoted by $(b)$ in (40), 449 we note that both $X^{\prime}$ and $Y$ are independent and non40 identically distributed lognormal random variables, and approximate the distribution of $X^{\prime}+Y$ by another $\log$ normal distribution, denoted by $Z$ [46]. That is, we let $Z \sim \mathcal{L N}\left(\mu_{z}, \sigma_{z}^{2}\right)$. Following the method discussed in [46], the parameters $\mu_{z}$ and $\sigma_{z}^{2}$ are calculated as

$$
\mu_{z}=\frac{1}{2} \log \mathbb{E}\left[Z^{-1}\right]-2 \log \mathbb{E}\left[Z^{-2}\right],
$$

$$
\sigma_{z}^{2}=\log \mathbb{E}\left[Z^{-2}\right]-2 \log \mathbb{E}\left[Z^{-1}\right] .
$$

where the moments $\mathbb{E}\left[Z^{-1}\right]$ and $\mathbb{E}\left[Z^{-2}\right]$ are either obtained through simulations, or numerically estimated as

$$
\begin{aligned}
& \mathbb{E}\left[Z^{-1}\right]=\int_{0}^{\infty} \int_{0}^{\infty}(x+y)^{-1} f_{X^{\prime}}(x) f_{Y}(y) d x d y, \\
& \mathbb{E}\left[Z^{-2}\right]=\int_{0}^{\infty} \int_{0}^{\infty}(x+y)^{-2} f_{X^{\prime}}(x) f_{Y}(y) d x d y,
\end{aligned}
$$

where $f_{X^{\prime}}(x)$ and $f_{Y}(y)$ are the PDFs of the lognormal random variables $X^{\prime}$ and $Y$. Following these approximations, the outage probability at $\mathrm{D}$ is given as

$$
P_{\text {out }}^{(D, 2)}=\frac{1}{2} \operatorname{erfc}\left(-\frac{\log \left(\gamma_{2}\right)-\mu_{z}}{\sqrt{2} \sigma_{z}}\right) .
$$

As mentioned earlier, we establish the tightness of the derived expression (46) later in Sec. V.

Now, we define the probability of overall outage at D. Recall that we assume that $\mathrm{D}$ decodes $s_{1}$ in the first time slot successfully. For case C1, the overall outage covers the scenario when $D$ fails to decode $s_{2}$ either over the RD or SD links, and is defined as

$$
P_{\text {out }}^{(1)} \triangleq P_{\text {out }}^{(R)} P_{\text {out }}^{(D, 1)} .
$$

Similarly, it is easy to see that the overall outage probability in case $\mathrm{C} 2$ is governed by the scenario that $\mathrm{D}$ fails to decode $s_{2}$ if either R fails to decode $s_{1}$ over the SR link or D fails to decode $s_{2}$ over the RD link, and is given by

$$
P_{\text {out }}^{(2)} \triangleq P_{\text {out }}^{(R)}+\left(1-P_{\text {out }}^{(R)}\right) P_{\text {out }}^{(D, 2)} \text {. }
$$

\section{2) IMPERFECT DECODING OF $s_{1}$ AT D}

Next, we relax the assumption of perfect decoding of $s_{1}$ at D. If $\mathrm{D}$ does not decode $s_{1}$ in the first time slot, then the overall outage probability is given by

$$
P_{\text {out }}^{(S S)}=\operatorname{Pr}\left\{\mathcal{U}<\gamma_{1}, \mathcal{V}<\gamma_{2}\right\},
$$

where

$$
\begin{aligned}
& \mathcal{U} \triangleq \min \left\{\zeta_{S}^{\left(s_{1}\right)}, \zeta_{D}^{\left(s_{1}\right)}\right\}, \quad \text { and } \\
& \mathcal{V} \triangleq \min \left\{\zeta_{S}^{\left(s_{2}\right)}, \zeta_{D}^{\left(s_{2}, 1\right)}\right\} .
\end{aligned}
$$

In order to obtain the closed form solution for the CDF in (49), we consider the following approximation. Since the end-to-end capacity of a decode and forward-based relaying network is determined by the weakest link capacity, we assume that $\min \left\{\zeta_{S}^{\left(s_{1}\right)}, \zeta_{D}^{\left(s_{1}\right)}\right\} \approx \zeta_{D}^{\left(s_{1}\right)}$, which corresponds to the link between the S-D as D is always placed far from $\mathrm{S}$ compared to $\mathrm{R}$. Similarly, we assume that $\min \left\{\zeta_{S}^{\left(S_{2}\right)}, \zeta_{D}^{\left(S_{2}, 1\right)}\right\} \approx \zeta_{D}^{\left(s_{2}, 1\right)}$, which implies that $\mathcal{U}=\zeta_{D}^{\left(S_{1}\right)}$ and $\mathcal{V}=\zeta_{D}^{\left(s_{2}, 1\right)}$. In Section $\mathrm{V}$, we show that this approximation is tight, through Monte Carlo simulations. Therefore, the outage probability at $\mathrm{D}$ when $s_{1}$ is not decoded perfectly is given by

$$
P_{\text {out }}^{(S S)}=\frac{1}{2} \operatorname{erfc}\left(-\frac{\log (v)-2 \mu}{\sqrt{8} \sigma}\right),
$$




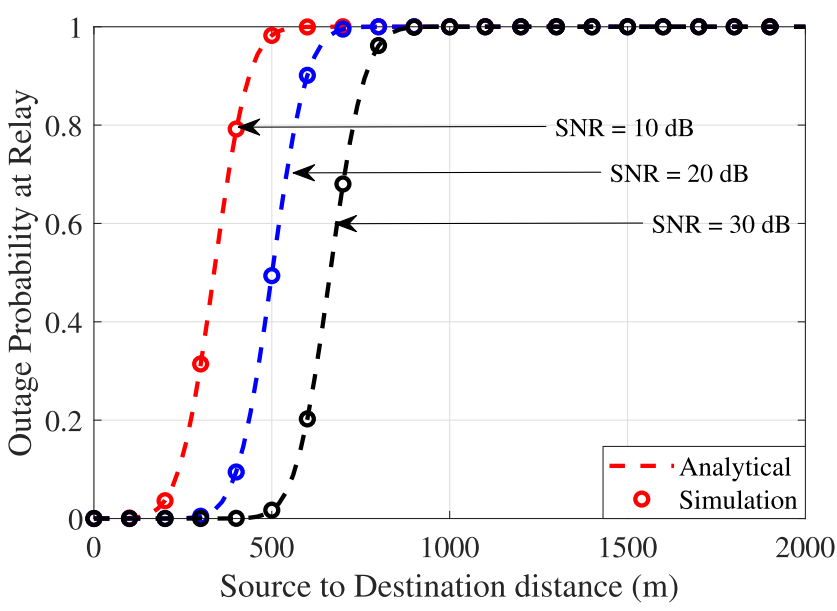

FIGURE 2. Outage probability at the relay for different SNRs.

where $v=\max \left(\frac{\gamma_{1}}{\left(b_{1}-\gamma_{1} b_{2}\right) G_{S D} \rho}, \frac{\gamma_{2}}{\left(b_{2}-\gamma_{2} b_{1}\right) G_{S D} \rho}\right)$. Next, 500 we study the outage probability analysis for DS NOMA.

\section{B. DS NOMA}

${ }_{02}$ The overall outage probability for the DS NOMA is given by

504 where

$$
P_{\text {out }}^{(D S)}=\operatorname{Pr}\left\{\mathcal{A}<\gamma_{1}, \mathcal{B}<\gamma_{2}\right\},
$$

$$
\begin{aligned}
& \mathcal{A}=\min \left\{\zeta_{S}^{\left(S_{1}\right)}, \zeta_{D}^{(I)}\right\}, \quad \text { and } \\
& \mathcal{B}=\min \left\{\zeta_{S}^{\left(S_{2}\right)}, \zeta_{D}^{(I I)}\right\} .
\end{aligned}
$$

Obtaining the closed-form expression for (53) is hard, as it involves correlated random variables $\mathcal{A}$ and $\mathcal{B}$. Towards this end, we employ an approximation similar to what was used in Section III-A2. Since the end-to-end capacity of a decode and forward relay-based system is determined by the weakest link capacity, we assume that $\min \left\{\zeta_{S}^{\left(s_{1}\right)}, \zeta_{D}^{(I)}\right\} \approx \zeta_{D}^{(I)}$, which corresponds to the link between S-D as D is always placed far from $S$ in comparison with R. Similarly, $\min \left\{\zeta_{S}^{\left(s_{2}\right)}, \zeta_{D}^{(I I)}\right\} \approx$ $\zeta_{D}^{(I I)}$. Therefore $\mathcal{A}=\zeta_{D}^{(I)}$ and $\mathcal{B}=\zeta_{D}^{(I I)}$. Once again, the tightness of this approximation is validated through Monte Carlo simulations in Section V. Next, we consider the problem of optimal power allocation for both SS NOMA and DS NOMA schemes, formulate the corresponding optimization problems and discuss their solutions.

\section{OPTIMAL POWER ALLOCATION}

In this section, we consider the problem of optimal power allocation at $\mathrm{S}$, such that the probability of overall outage is minimized.

\section{A. SS NOMA}

We formulate the following optimization problems for both cases $\mathrm{C} 1$ and $\mathrm{C} 2$ as

$$
\begin{aligned}
& \min _{b_{1}} P_{\text {out }}^{(R)} P_{\text {out }}^{(D, 1)} \\
& \text { s.t. } 0<b_{1}<1,
\end{aligned}
$$

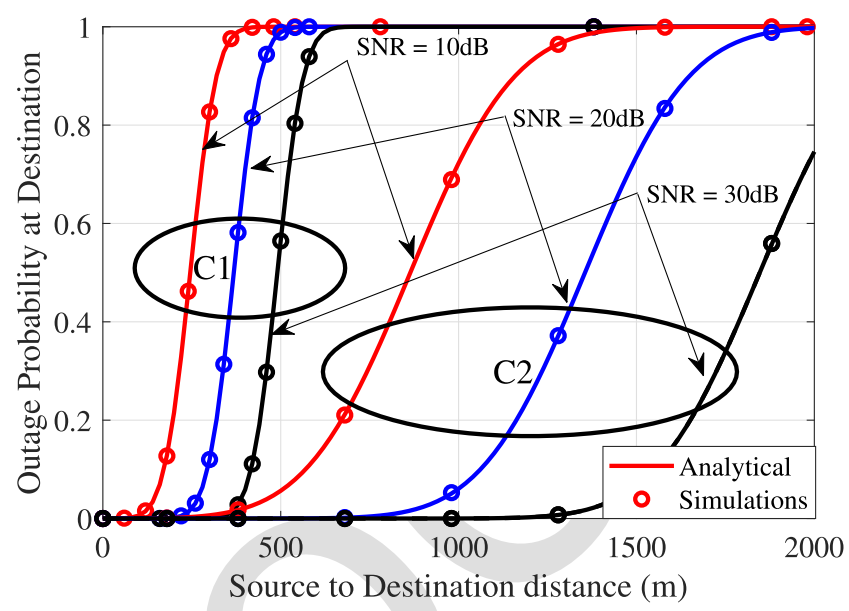

FIGURE 3. Outage probability at the destination for different SNRs.

and

$$
\begin{aligned}
& \min _{b_{1}} P_{\text {out }}^{(R)}+\left(1-P_{\text {out }}^{(R)}\right) P_{\text {out }}^{(D, 2)} \\
& \text { s.t. } 0<b_{1}<1,
\end{aligned}
$$

respectively. The solution of the above optimization problems for both cases is described in the following proposition; this interestingly shows that both cases have the same solution.

Proposition 1: The solution to the optimization problem given in (56) for both cases $\mathrm{C} 1$ and $\mathrm{C} 2$ is

$$
b_{1}^{*}=\frac{\gamma_{1}\left(1+\gamma_{2}\right)}{\gamma_{1}\left(1+\gamma_{1}\right)+\gamma_{2}} \text {. }
$$

Proof: See Appendix A.$$
530
$$

\section{B. DS NOMA}

In case of DS NOMA, the optimization will be jointly over the power allocation coefficients $\left\{b_{1}, b_{2}\right\}$ and $\left\{b_{3}, b_{4}\right\}$. Therefore, the optimization problem in this case is formulated as

$$
\begin{aligned}
& \min _{b_{1}, b_{4}} P_{\text {out }}^{(D S)} \\
& \text { s.t. } 0<b_{1}<1, \\
& 0<b_{4}<1 .
\end{aligned}
$$

The solution of the above optimization problems is described in the following proposition.

Proposition 2: The solution to the optimization problem given in (60) for DS NOMA is

$$
b_{1}^{*}=b_{4}^{*}=\frac{\left(\gamma_{2} G_{R D}^{2}-\gamma_{1} G_{S D}^{2}\right)}{\left(\gamma_{1} G_{R D}^{2}-\gamma_{1} G_{S D}^{2}-\gamma_{2} G_{S D}^{2}+\gamma_{2} G_{R D}^{2}\right)} .
$$

Proof: See Appendix B.

\section{RESULTS AND DISCUSSION}

In this section, we validate our analysis on outage probability through Monte Carlo simulations and numerical techniques. The set of parameters chosen for our study are as follows. The PLC cable attenuation model is considered to be $G_{i}\left(v_{i}, f\right)=$ $\exp \left(-\beta v_{i}\right)$, for $i \in\{\mathrm{SD}, \mathrm{SR}, \mathrm{RD}\}$, where $\beta=d_{0}+d_{1} f^{m}$ is the 


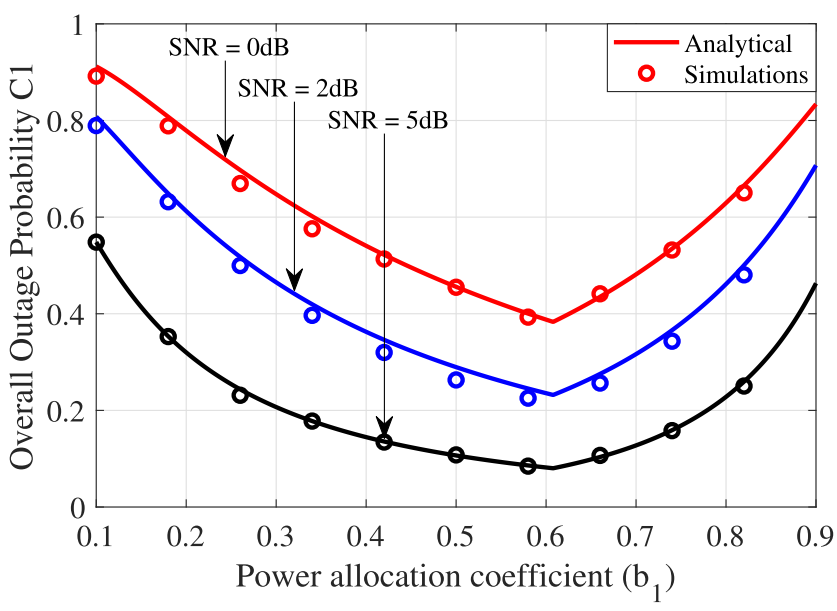

FIGURE 4. Outage probability for different $b_{1}$ at different SNRs, without MRC.

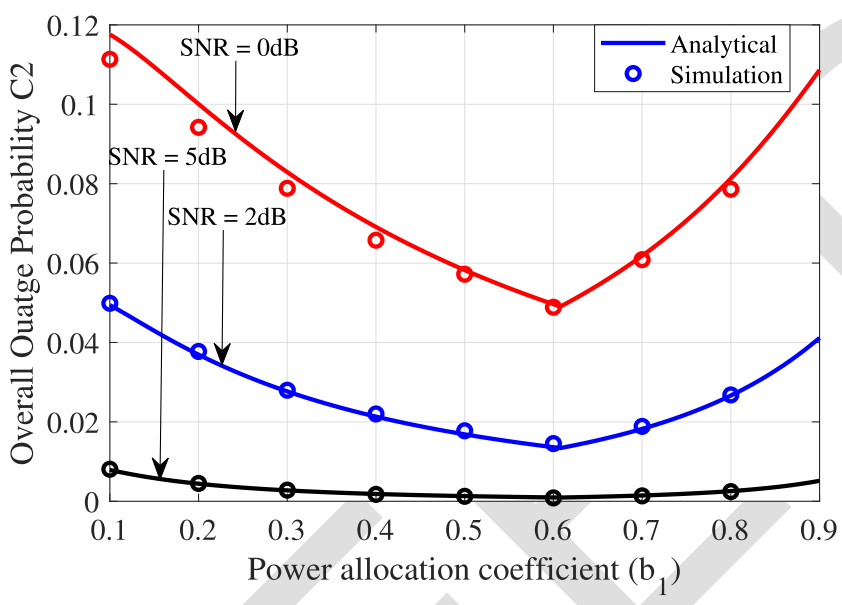

FIGURE 5. Outage probability for different $b_{1}$ at different SNRs, with MRC.

$\mu=1.5 \mathrm{~dB}, \sigma=5 \mathrm{~dB}[32]$, and $v_{S R}=\frac{1}{4} v_{S D}$. Additionally

${ }_{564}=1 / 30$, and $\gamma_{2}=1 / 20$.

Figure 2 shows the variation of the outage probability at $\mathrm{R}$, that is, $P_{\text {out }}^{(R)}$ as a function of the distance from S. First, the plots establish the perfect match between our analysis and Monte Carlo simulations. Next, observe that for a given SNR, $P_{\text {out }}^{(R)}$ increases with an increase in the distance between S-R, as expected. Variation of the outage probability at $\mathrm{D}$ with the distance between S-D for the two cases C1 and C2 are shown in Fig. 3. As expected, the loss in outage for $\mathrm{C} 1$ is significantly severe as compared to that for $\mathrm{C} 2$, especially at high SNRs. Moreover, the excellent agreement between our analysis and Monte Carlo simulations establishes the high accuracy of our simple two-tier approximation.

Figure 4 shows the variation of overall probability of outage, $P_{\text {out }}$, as a function of the power allocation coefficient $579 b_{1}$, for different SNR values in case C1, without MRC.

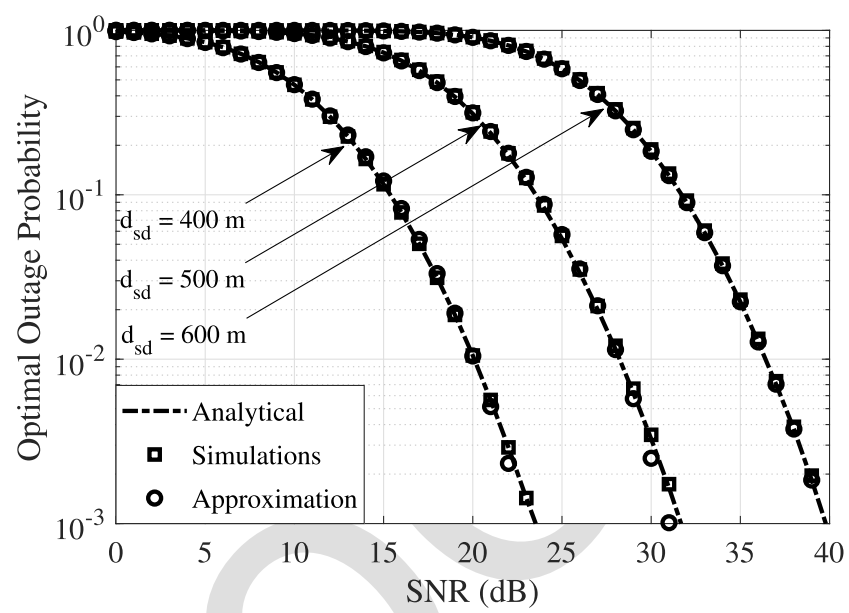

FIGURE 6. Overall outage probability of the SS NOMA for different SNRs and end-to-end distances. Here, $\mu=3 \mathrm{~dB}$.

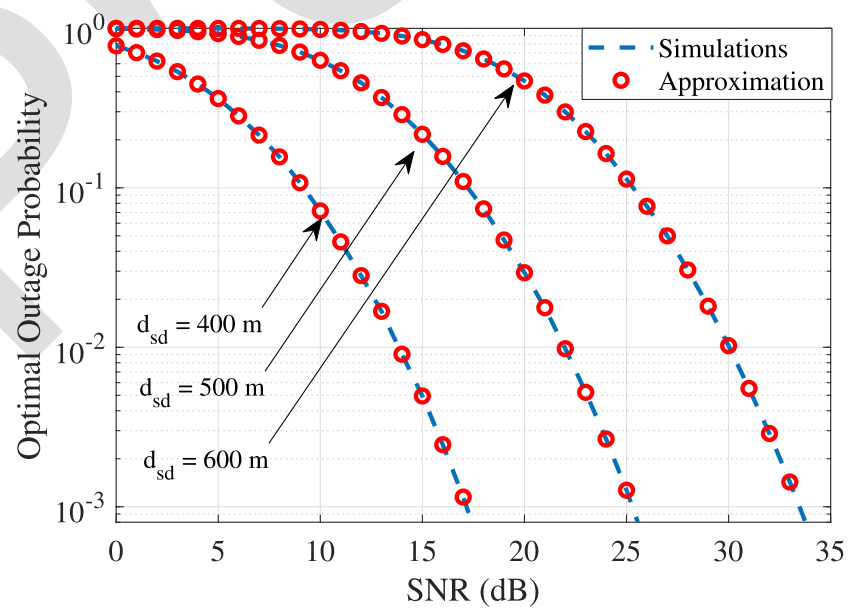

FIGURE 7. Overall outage probability of the DS NOMA for different SNRs and end-to-end distances. Here, $\mu=3 \mathrm{~dB}$.

As expected, $P_{\text {out }}$ is convex in the range of $b_{1} \in(0,1)$, and the optimal occurs at $b_{1}^{*}$ given in (58). Additionally, note that $b_{1}^{*}$ depends only on the values of $\gamma_{1}$ and $\gamma_{2}$. Moreover, the plots obtained through numerical techniques and simulations are in close agreement, thereby validating our analysis. A similar set of conclusions can be drawn from Fig. 5, where the variation of $P_{\text {out }}$ is given for case $\mathrm{C} 2$, for the same set of parameters chosen for Fig. 4. Note that although employing MRC at the destination for $\mathrm{C} 2$ significantly improves the performance in terms of the outage probability, the optimal $b_{1}$ does not change. Therefore, the design of optimal $b_{1}$ for the considered system model does not depend on whether $\mathrm{R}$ decodes $s_{2}$ successfully or not.

Figures 6 and 7 show the variation of optimal outage probabilities of SS NOMA and DS NOMA with SNR, respectively. First, the perfect match between our analysis and Monte Carlo simulations establish the validity of our derived theoretical expressions, and the accuracy of our approximations considered in Sections III-A2 and III-B. Second, following these plots, it is obvious that the SNR required for DS NOMA 


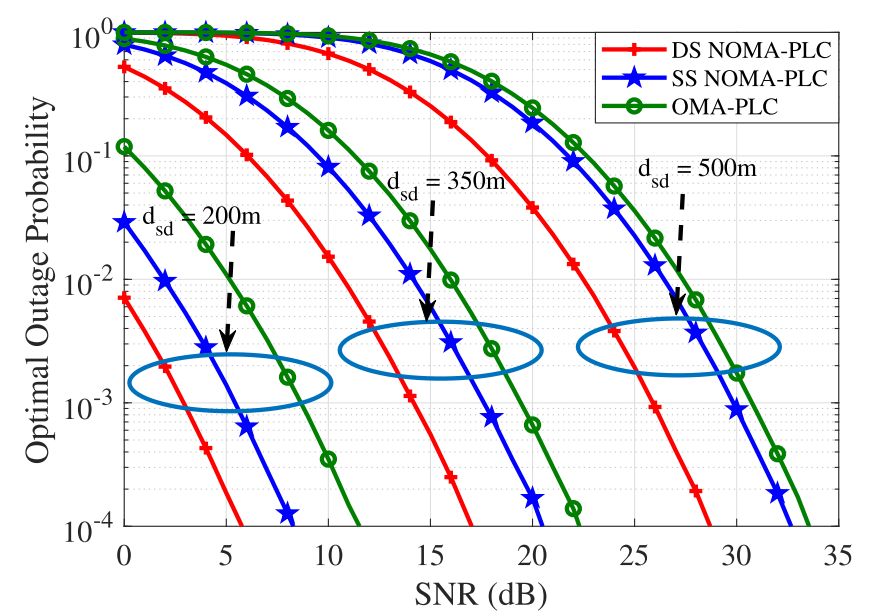

FIGURE 8. Comparison of overall outage probabilities of DS NOMA, SS NOMA and OMA schemes, for different SNRs and end-to-end distances. It is assumed that $\mu=3 \mathrm{~dB}$. scheme to achieve a given outage probability will be considerably lower compared to that of the SS NOMA scheme. Moreover, it was observed that the performance of both relaybased schemes improves with an increase in parameter $\mu$ [1]. However, the performances of both schemes deteriorate with an increase in $\sigma^{2}$, and as the distance between $\mathrm{S}$ and $\mathrm{D}$ increases [47].

Figure 8 shows the comparison of the overall outage probabilities for SS NOMA, DS NOMA and OMA schemes. As expected, it is seen that the DS NOMA scheme outperforms the SS NOMA scheme for all the considered system configurations. Additionally, note that as the SNR increases, the overall outage probability decreases and more so when the channel variances are relatively small. A NOMA-based PLC system is also known to outperform an OMA-based PLC system in terms of spectral efficiency, as noted in [32].

\section{CONCLUSION AND FUTURE WORK}

We presented a detailed study on the performance of NOMA-aided, relay-based cooperative communication in PLC networks, in terms of the overall probability of outage. We derived closed form expressions for the outage probabilities at the relay and the destination, for SS and DS NOMA schemes. In particular, the outage probabilities at the destination for SS-NOMA and DS-NOMA were found out using approximations, which were shown to be tight using numerical techniques. Next, we formulated optimization problems to find optimal power allocation coefficients at the source for SS NOMA and at the relay for DS NOMA, and derived closed form solutions for the corresponding optimal power allocation coefficients. Between the two, we showed that DS NOMA outperforms SS NOMA, in terms of outage probability. Further, we established the accuracy of our analysis through Monte Carlo simulations.

Some of the key assumptions in this work include performance analysis under Gaussian noise - which is an information-theoretic worst-case scenario, and perfect SIC at both $\mathrm{R}$ and $\mathrm{D}$. However, the impulsive noise has been observed to be prominent component that severely affects the performance of a PLC system, which can be mitigated by the use of techniques such as nulling and waveform clipping [18]. Additionally, a study on the impact of imperfect SIC on the performance of the system would be interesting to investigate. Moreover, we have not considered a MIMO setup, which is known to improve the performance of a PLC system. A detailed study of these topics are reserved for our future work in this direction.

\section{APPENDIX A}

\section{PROOF OF PROPOSITION 1}

First, consider case $\mathrm{C} 1$. We provide an outline of the proof and exclude some algebraic details that lead to lengthy expressions for brevity. Note that $b_{1}^{*}$ is obtained by equating the terms inside the $\max (\cdot, \cdot)$ term in the definition of $\Gamma$. From (56), it can be shown that the derivative of the cost function

$$
\begin{aligned}
\frac{d P_{\text {out }}}{d b_{1}}= & \frac{1}{2 \sigma \sqrt{8 \pi}}\left\{\operatorname{erfc}\left(\frac{K_{S R}}{\sqrt{8} \sigma}\right) \exp \left(-\left(\frac{K_{S D}}{\sqrt{8} \sigma}\right)^{2}\right)\right. \\
& \times\left(\frac{-1-\gamma_{2}}{\left(\gamma_{2}+1\right) b_{1}-1}\right)+\left(\frac{-1-\gamma_{1}}{b_{1}\left(\gamma_{1}+1\right)-\gamma_{1}}\right) \\
& \left.\times \operatorname{erfc}\left(\frac{K_{S D}}{\sqrt{8} \sigma}\right) \exp \left(-\left(\frac{K_{S R}}{\sqrt{8} \sigma}\right)^{2}\right)\right\}<0,
\end{aligned}
$$

where

$$
\begin{aligned}
& K_{S R} \triangleq-\log (\Gamma)-\left(2 \mu+\log \left(P\left[G_{S R}\left(v_{S R}, f\right)\right]^{2}\right)\right), \quad \text { and } \\
& K_{S D} \triangleq \log \left(\gamma_{2} \sigma_{d}^{2}\right)-\left(2 \mu+\log \left(P\left[G_{S D}\left(v_{S D}, f\right)\right]^{2} \mathcal{K}\right)\right), \quad
\end{aligned}
$$

and monotone for all $b_{1} \in\left(\frac{\gamma_{1}\left(1+\gamma_{2}\right)}{\gamma_{1}\left(1+\gamma_{2}\right)+\gamma_{2}}, 1\right]$. Similarly, the derivative for all $b_{1} \in\left[0, \frac{\gamma_{1}\left(1+\gamma_{2}\right)}{\gamma_{1}\left(1+\gamma_{2}\right)+\gamma_{2}}\right)$ can be shown to be

$$
\begin{aligned}
\frac{d P_{\text {out }}}{d b_{1}}= & \frac{1}{2 \sigma \sqrt{8 \pi}}\left\{\operatorname{erfc}\left(\frac{K_{S R}}{\sqrt{8} \sigma}\right) \exp \left(-\left(\frac{K_{S D}}{\sqrt{8} \sigma}\right)^{2}\right)\right. \\
& \times\left(\frac{-1-\gamma_{2}}{\left(\gamma_{2}+1\right) b_{1}-1}\right)+\left(\frac{1}{1-b_{1}}\right) \\
& \left.\times \operatorname{erfc}\left(\frac{K_{S D}}{\sqrt{8} \sigma}\right) \exp \left(-\left(\frac{K_{S R}}{\sqrt{8} \sigma}\right)^{2}\right)\right\}>0,
\end{aligned}
$$

which is monotone for $b_{1} \in\left[0, \frac{\gamma_{1}\left(1+\gamma_{2}\right)}{\gamma_{1}\left(1+\gamma_{2}\right)+\gamma_{2}}\right)$. Therefore, the solution for optimal $b_{1}$ is when both the derivatives are equal, which gives the required $b_{1}^{*}$.

The proof for case $\mathrm{C} 2$ is similar. From (56), it can be shown that the derivative of the cost functions are

$$
\begin{aligned}
\frac{d P_{\text {out }}}{d b_{1}}= & \frac{1}{\sqrt{8 \pi} \sigma} \exp \left(-\left(\frac{K_{S R}}{\sqrt{8} \sigma}\right)^{2}\right)\left(\frac{-1-\gamma_{1}}{b_{1}\left(\gamma_{1}+1\right)-\gamma_{1}}\right) \\
& +\frac{1}{\sqrt{8 \pi} \sigma} \exp \left(-\left(\frac{K_{S D}}{\sqrt{8} \sigma}\right)^{2}\right)\left(\frac{-1-\gamma_{2}}{\left(\gamma_{2}+1\right) b_{1}-1}\right)
\end{aligned}
$$




$$
\begin{aligned}
& -\frac{1}{2 \sigma \sqrt{8 \pi}}\left\{\operatorname{erfc}\left(\frac{K_{S R}}{\sqrt{8} \sigma}\right) \exp \left(-\left(\frac{K_{S D}}{\sqrt{8} \sigma}\right)^{2}\right)\right. \\
& \times\left(\frac{-1-\gamma_{2}}{\left(\gamma_{2}+1\right) b_{1}-1}\right)+\left(\frac{-1-\gamma_{1}}{b_{1}\left(\gamma_{1}+1\right)-\gamma_{1}}\right) \\
& \left.\times \operatorname{erfc}\left(\frac{K_{S D}}{\sqrt{8} \sigma}\right) \exp \left(-\left(\frac{K_{S R}}{\sqrt{8} \sigma}\right)^{2}\right)\right\}<0,
\end{aligned}
$$

675 and monotone for all $b_{1} \in\left(\frac{\gamma_{1}\left(1+\gamma_{2}\right)}{\gamma_{1}\left(1+\gamma_{2}\right)+\gamma_{2}}, 1\right]$. Similarly,

676 the derivative for all $b_{1} \in\left[0, \frac{\gamma_{1}\left(1+\gamma_{2}\right)}{\gamma_{1}\left(1+\gamma_{2}\right)+\gamma_{2}}\right)$

${ }_{677} \frac{d P_{\text {out }}}{d b_{1}}=\frac{1}{\sqrt{8 \pi} \sigma} \exp \left(-\left(\frac{K_{S R}}{\sqrt{8} \sigma}\right)^{2}\right)\left(\frac{1}{1-b_{1}}\right)$$$
+\frac{1}{\sqrt{8 \pi} \sigma} \exp \left(-\left(\frac{K_{S D}}{\sqrt{8} \sigma}\right)^{2}\right)\left(\frac{-1-\gamma_{2}}{\left(\gamma_{2}+1\right) b_{1}-1}\right)
$$$$
-\frac{1}{2 \sigma \sqrt{8 \pi}}\left\{\operatorname{erfc}\left(\frac{K_{S R}}{\sqrt{8} \sigma}\right) \exp \left(-\left(\frac{K_{S D}}{\sqrt{8} \sigma}\right)^{2}\right)\right.
$$$$
\times\left(\frac{-1-\gamma_{2}}{\left(\gamma_{2}+1\right) b_{1}-1}\right)+\left(\frac{1}{1-b_{1}}\right)
$$$$
\left.\times \operatorname{erfc}\left(\frac{K_{S D}}{\sqrt{8} \sigma}\right) \exp \left(-\left(\frac{K_{S R}}{\sqrt{8} \sigma}\right)^{2}\right)\right\}>0,
$$

${ }_{682}$ and monotone for $b_{1} \in\left[0, \frac{\gamma_{1}\left(1+\gamma_{2}\right)}{\gamma_{1}\left(1+\gamma_{2}\right)+\gamma_{2}}\right)$.

\section{APPENDIX B}

\section{PROOF OF PROPOSITION 2}

We follow an approach similar to that used in the proof of Proposition 1, discussed in Appendix VI. The proof involves in first finding the first partial derivative of the cost function in (60) with respect to $b_{4}$ to establish the convexity of the cost in (60) through monotonicity arguments similar to the development in Appendix VI. Later, it is shown that the minimum is obtained at the corner point, given by $b_{4}^{*}$ given in (61). These steps are repeated by evaluating partial derivatives with respect to $b_{1}$ to obtain $b_{1}^{*}$ The corresponding analysis, although straightforward, leads to lengthy mathematical expressions, which are omitted for brevity.

\section{REFERENCES}

[1] R. Ramesh, S. Gurugopinath, and S. Muhaidat, "Outage performance of relay-assisted NOMA over power line communications," in Proc. IEEE 31st Annu. Int. Symp. Pers., Indoor Mobile Radio Commun., Aug. 2020, pp. 1-6.

[2] Y. Kim, J. Bae, and J. Kim, "Performance of power line communication systems with noise reduction scheme for smart grid applications," IEEE Trans. Consum. Electron., vol. 57, no. 1, pp. 46-52, Feb. 2011.

[3] M. Zimmermann and K. Dostert, "A multipath model for the powerline channel," IEEE Trans. Commun., vol. 50, no. 4, pp. 553-559, Apr. 2002.

[4] M. Rozman, A. Ikpehai, B. Adebisi, and K. M. Rabie, "Channel characterisation of cooperative relaying power line communication systems," in Proc. 10th Int. Symp. Commun. Syst., Netw. Digit. Signal Process. (CSNDSP), Jul. 2016, pp. 1-5.

[5] L. Di Bert, P. Caldera, D. Schwingshackl, and A. M. Tonello, "On noise modeling for power line communications," in Proc. IEEE Int. Symp. Power Line Commun. Appl., Apr. 2011, pp. 283-288.

[6] A. M. Tonello and A. Pittolo, "Considerations on narrowband and broadband power line communication for smart grids," in Proc. SmartGridComm, Nov. 2015, pp. 13-18.
[7] A. Ikpehai, B. Adebisi, and K. Rabie, "Broadband PLC for clustered advanced metering infrastructure (AMI) architecture," Energies, vol. 9, no. 7, p. 569, Jul. 2016, doi: 10.3390/en9070569.

[8] S. Galli, A. Scaglione, and Z. Wang, "For the grid and through the grid: The role of power line communications in the smart grid," Proc. IEEE, vol. 99, no. 6, pp. 998-1027, Jun. 2011

[9] A. Ikpehai, B. Adebisi, K. Rabie, R. Haggar, and M. Baker, "Experimental study of 6LoPLC for home energy management systems," Energies, vol. 9 , no. 12 , p. 1046 , Dec. 2016, doi: 10.3390/en9121046.

[10] D. Righini, F. Passerini, and A. M. Tonello, "Modeling transmission and radiation effects when exploiting power line networks for communication," IEEE Trans. Electromagn. Compat., vol. 60, no. 1, pp. 59-67, Feb. 2018.

[11] A. Pittolo and A. M. Tonello, "A synthetic statistical MIMO PLC channel model applied to an in-home scenario," IEEE Trans. Commun., vol. 65, no. 6, pp. 2543-2553, Jun. 2017.

[12] H. Pu, X. Liu, S. Zhang, and D. Xu, "Adaptive cooperative non-orthogonal multiple access-based power line communication," IEEE Access, vol. 7 , pp. 73856-73869, 2019.

[13] M. Nassar, J. Lin, Y. Mortazavi, A. Dabak, I. H. Kim, and B. L. Evans, "Local utility power line communications in the 3-500 kHz band: Channel impairments, noise, and standards," IEEE Signal Process. Mag., vol. 29, no. 5, pp. 116-127, Sep. 2012.

[14] K. M. Rabie and E. Alsusa, "Effective noise cancellation using singlecarrier FDMA transmission in power-line channels," IEEE Trans. Power Del., vol. 29, no. 5, pp. 2110-2117, Oct. 2014.

[15] R. Pighi, M. Franceschini, G. Ferrari, and R. Raheli, "Fundamental performance limits of communications systems impaired by impulse noise," IEEE Trans. Commun., vol. 57, no. 1, pp. 171-182, Jan. 2009.

[16] B. Adebisi and B. Honary, "Comparisons of indoor PLC emissions measurement results and regulation standards," in Proc. IEEE Int. Symp. Power Line Commun. Appl., Mar. 2006, pp. 319-324.

[17] H. Meng, S. Chen, Y. L. Guan, C. L. Law, P. L. So, E. Gunawan, and T. T. Lie, "Modeling of transfer characteristics for the broadband power line communication channel," IEEE Trans. Power Del., vol. 19, no. 3, pp. 1057-1064, Jul. 2004

[18] K. M. Rabie and E. Alsusa, "Effective noise cancellation using singlecarrier FDMA transmission in power-line channels," IEEE Trans. Power Del., vol. 29, no. 5, pp. 2110-2117, Oct. 2014.

[19] J. Lin, M. Nassar, and B. L. Evans, "Impulsive noise mitigation in powerline communications using sparse Bayesian learning," IEEE J. Sel. Areas Commun., vol. 31, no. 7, pp. 1172-1183, Jul. 2013.

[20] J. Lin, T. Pande, I. H. Kim, A. Batra, and B. L. Evans, "Time-frequency modulation diversity to combat periodic impulsive noise in narrowband powerline communications," IEEE Trans. Commun., vol. 63, no. 5, pp. 1837-1849, May 2015.

[21] I. H. Kim, B. Varadarajan, and A. Dabak, "Performance analysis and enhancements of narrowband OFDM powerline communication systems," in Proc. 1st IEEE Int. Conf. Smart Grid Commun., Oct. 2010, pp. 362-367.

[22] W. Duan, J. Ju, J. Hou, Q. Sun, X.-Q. Jiang, and G. Zhang, "Effective resource utilization schemes for Decode-and-Forward relay networks with NOMA," IEEE Access, vol. 7, pp. 51466-51474, 2019.

[23] M. K. Shukla, H. H. Nguyen, and O. J. Pandey, "Secrecy performance analysis of two-way relay non-orthogonal multiple access systems," IEEE Access, vol. 8, pp. 39502-39512, 2020.

[24] L. Lampe and A. J. H. Vinck, "Cooperative multihop power line communications," in Proc. IEEE Int. Symp. Power Line Commun. Appl., Mar. 2012, pp. $1-6$.

[25] A. M. Tonello, F. Versolatto, and S. D'Alessandro, "Opportunistic relaying in in-home PLC networks," in Proc. IEEE Global Telecommun. Conf. (GLOBECOM), Dec. 2010, pp. 1-5.

[26] X. Cheng, R. Cao, and L. Yang, "Relay-aided amplify-and-forward powerline communications," IEEE Trans. Smart Grid, vol. 4, no. 1, pp. 265-272, Mar. 2013.

[27] K. M. Rabie, B. Adebisi, and A. Salem, "Improving energy efficiency in dual-hop cooperative PLC relaying systems," in Proc. Int. Symp. Power Line Commun. Appl. (ISPLC), Mar. 2016, pp. 196-200.

[28] A. Benjebbour, A. Li, Y. Saito, Y. Kishiyama, A. Harada, and T. Nakamura "System-level performance of downlink NOMA for future LTE enhancements," in Proc. IEEE Globecom Workshops (GC Wkshps), Dec. 2013 , pp. 66-70.

[29] Y. Lan, A. Benjebbour, A. Li, and A. Harada, "Efficient and dynamic fractional frequency reuse for downlink non-orthogonal multiple access," in Proc. IEEE 79th Veh. Technol. Conf. (VTC Spring), May 2014, pp. 1-5. 
[30] Z. Ding, Z. Yang, P. Fan, and H. V. Poor, "On the performance of nonorthogonal multiple access in $5 \mathrm{G}$ systems with randomly deployed users,' IEEE Signal Process. Lett., vol. 21, no. 12, pp. 1501-1505, Dec. 2014.

[31] J. Choi, "Non-orthogonal multiple access in downlink coordinated twopoint systems," IEEE Commun. Lett., vol. 18, no. 2, pp. 313-316, Feb. 2014

[32] K. M. Rabie, B. Adebisi, E. H. G. Yousif, H. Gacanin, and A. M. Tonello, "A comparison between orthogonal and non-orthogonal multiple access in cooperative relaying power line communication systems," IEEE Access, vol. 5, pp. 10118-10129, 2017.

[33] X. Liang, Y. Wu, D. W. K. Ng, S. Jin, Y. Yao, and T. Hong, "Outage probability of cooperative NOMA networks under imperfect CSI with user selection," IEEE Access, vol. 8, pp. 117921-117931, 2020.

[34] D.-T. Do, T.-T.-T. Nguyen, T. N. Nguyen, X. Li, and M. Voznak, "Uplink and downlink NOMA transmission using full-duplex UAV," IEEE Access, vol. 8, pp. 164347-164364, 2020.

[35] S. M. R. Islam, N. Avazov, O. A. Dobre, and K.-S. Kwak, "Power-domain non-orthogonal multiple access (NOMA) in 5G systems: Potentials and challenges," IEEE Commun. Surveys Tuts., vol. 19, no. 2, pp. 721-742, 2nd Quart., 2017.

[36] X. Xie, J. Liu, J. Huang, and S. Zhao, "Ergodic capacity and outage performance analysis of uplink full-duplex cooperative NOMA system," IEEE Access, vol. 8, pp. 164786-164794, 2020.

[37] J. Ju, W. Duan, Q. Sun, S. Gao, and G. Zhang, "Performance analysis for cooperative NOMA with opportunistic relay selection," IEEE Access, vol. 7, pp. 131488-131500, 2019.

[38] Y. Liu, Z. Ding, M. Elkashlan, and H. V. Poor, "Cooperative nonorthogonal multiple access with simultaneous wireless information and power transfer," IEEE J. Sel. Areas Commun., vol. 34, no. 4, pp. 938-953, Apr. 2016.

[39] X. Yue, Y. Liu, S. Kang, A. Nallanathan, and Z. Ding, "Exploiting full/halfduplex user relaying in NOMA systems," IEEE Trans. Commun., vol. 66 , no. 2, pp. 560-575, Feb. 2018

[40] K. M. Rabie and B. Adebisi, "Enhanced amplify-and-forward relaying in non-Gaussian PLC networks," IEEE Access, vol. 5, pp. 4087-4094, 2017.

[41] K. M. Rabie, B. Adebisi, A. M. Tonello, S. Yarkan, and M. Ijaz, "Two-stage non-orthogonal multiple access over power line communication channels," IEEE Access, vol. 6, pp. 17368-17376, 2018.

[42] A. M. Tonello and F. Versolatto, "New results on top-down and bottomup statistical PLC channel modeling," in Proc. 3rd Workshop Power Line Commun. App., Oct. 2009, pp. 11-14.

[43] A. M. Tonello, F. Versolatto, and A. Pittolo, "In-home power line communication channel: Statistical characterization," IEEE Trans. Commun., vol. 62, no. 6, pp. 2096-2106, Jun. 2014.

[44] M. De Piante and A. M. Tonello, "On impedance matching in a powerline-communication system," IEEE Trans. Circuits Syst. II, Exp. Briefs, vol. 63, no. 7, pp. 653-657, Jul. 2016.

[45] X. Liang, X. Gong, Y. Wu, D. W. K. Ng, and T. Hong, "Analysis of outage probabilities for cooperative NOMA users with imperfect CSI," in Proc. IEEE 4th Inf. Technol. Mechatronics Eng. Conf. (ITOEC), Dec. 2018, pp. 1617-1623.

[46] J. C. S. S. Filho, P. Cardieri, and M. D. Yacoub, "Simple accurate lognormal approximation to lognormal sums," Electron. Lett., vol. 41, no. 18 , pp. 1016-1017, Sep. 2005.

[47] K. M. Rabie, B. Adebisi, and H. Gacanin, "Outage probability and energy efficiency of DF relaying power line communication networks: Cooperative and non-cooperative," in Proc. IEEE Int. Conf. Commun. (ICC), May 2017, pp. 1-6.

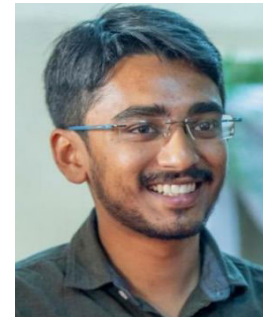

ROOPESH RAMESH (Graduate Student Member, IEEE) received the B.E. degree in electronics and communication engineering from the Dr. Ambedkar Institute of Technology, Visvesvaraya Technological University, Bengaluru, India, in 2016, and the M.Tech. degree in digita communication engineering from PES University, Bengaluru, in 2018, where he is currently pursuing the Ph.D. degree with the Department of Electronics and Communication Engineering. His current research interests include powerline communications, non-orthogonal multiple access (NOMA), and MIMO communications.

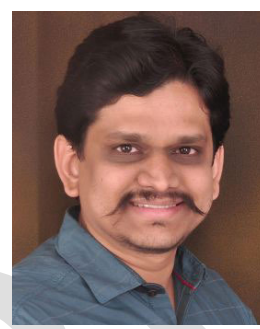

SANJEEV GURUGOPINATH (Member, IEEE) received the B.E. degree in electrical and electronics engineering from the Dr. Ambedkar Institute of Technology, Visvesvaraya Technological University, Bengaluru, India, in 2004, the M.Tech. degree in digital electronics and communication engineering from the M. S. Ramaiah Institute of Technology, Visvesvaraya Technological University, in 2006, and the $\mathrm{Ph} . \mathrm{D}$. degree in signal processing for communications from the Indian Institute of Science, Bengaluru, in 2015. He was a Visiting Research Scholar with Khalifa University, Abu Dhabi, United Arab Emirates. He is currently a Professor with the Department of Electronics and Communication Engineering, PES University, Bengaluru. His current research interests include cognitive radios for beyond $5 \mathrm{G}$ communication systems, powerline communications, underwater acoustics, and speech signal processing. He was a co-recipient of the Best Paper Awards at IEEE INDICON 2016, IEEE ICEECCOT 2019, and IEEE CONECCT 2020

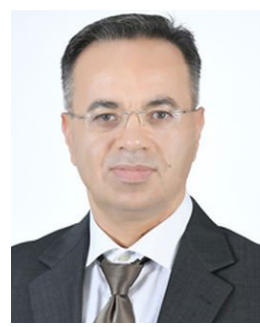

SAMI MUHAIDAT (Senior Member, IEEE) received the Ph.D. degree in electrical and computer engineering from the University of Waterloo, Waterloo, in 2006. From 2007 to 2008 he was an NSERC Postdoctoral Fellow with the Department of Electrical and Computer Engineering, University of Toronto, Canada. From 2008 to 2012, he was an Assistant Professor with the School of Engineering Science, Simon Fraser University, BC, Canada. He is currently a Professo with Khalifa University and an Adjunct Professor with Carleton University, Canada. His research interests include advanced digital signal processing techniques for wireless communications, RIS, 5G and beyond, MIMO, optical communications, the IoT with emphasis on battery-free devices, and machine learning. He served as a Senior Editor and an Editor for the IEEE Communications LetTers, an Editor of the IEEE Transactions on Communications, and an Associate Editor of the IEEE Transactions on Vehicular Technology. He is currently an Area Editor of the IEEE Transactions on Communications and a Lead Guest Editor of the IEEE Open Journal of the Communications Society (OJ-COMS) "Large-Scale Wireless Powered Networks with Backscatter Communications” Special Issue. 